\title{
POSTGRADO STRICTO SENSUS DEL DEPARTAMENTO DE ENFERMERÍA DE LA UNIVERSIDAD DE CONCEPCIÓN - CONCEPCIÓN, CHILE
}

Tatiana Paravic ${ }^{1}$

Paravic T. Postgrado stricto sensus del departamento de enfermería de la Universidad de Concepción - Concepción, Chile. Rev Latino-am Enfermagem 2004 novembro-dezembro; 12(6):946-53.

Artículo que relata la trayectoria e historia del Postgrado Stricto Sensus del Departamento de Enfermería de la Facultad de Medicina de la Universidad de Concepción. Concepción, Chile. Describe el diseño curricular de los programas , sus líneas de investigación, el producto que se quiere obtener y su impacto, así como el proceso para llegar a la aprobación del primer programa de Doctorado en Enfermería en Chile.

DESCRIPTORES: educación de postgrado en enfermería; enfermería

\section{STRICTO SENSU GRADUATE PROGRAM AT THE NURSING DEPARTMENT OF CONCEPCIÓN UNIVERSITY - CONCEPCIÓN, CHILE}

This article reports on the trajectory and history of the stricto sensu graduate program offered at the Nursing Department of Concepción University Medical School, Concepción, Chile. It describes the curricular design of its programs, its research lines, the desired product and its impact, as well as the process to arrive at the approval of the first Ph.D. program in nursing in chile.

DESCRIPTORS: education, nursing, graduate; nursing

\section{PÓS-GRADUAÇÃO STRICTO SENSU DO DEPARTAMENTO DE ENFERMAGEM DA UNIVERSIDAD DE CONCEPCIÓN - CONCEPCIÓN, CHILE}

Este artigo relata a trajetória histórica da Pós-Graduação stricto sensu do Departamento de Enfermagem da Faculdade de Medicina da Universidade de Concepción, Chile. Descreve o desenho curricular dos programas, suas linhas de investigação, o produto que se pretende obter e seu impacto, assim como o processo para a aprovação do primeiro programa de Doutorado em Enfermagem do Chile.

DESCRITORES: educação de pós-graduação em enfermagem; enfermagem

\footnotetext{
${ }^{1}$ Enfermera, Magister en Enfermería en Salud Comunitaria, Universidad de Concepción, Chile, Doutor en Enfermería pela Escuela de Enfermería de Ribeirão Preto, de la Universidad de São Paulo, Centro Colaborador de la OMS para el desarrollo de la investigación en enfermería, PostDirector Postgrado Departamento de Enfermería, Profesor Titular de los Programas de Pregrado, Especialización y Postgrado del Departamento de Enfermería de la Facultad de Medicina de la Universidad de Concepción - Chile, e-mail: tparavic@udec.cl
} 
INTRODUCCIÓN

La nueva configuración de la economía mundial, la revolución en materia de información y de las comunicaciones, el mercado de consumo y, la emergencia de nuevas culturas así como la visibilización internacional de culturas y subculturas que se encontraban latentes y/ o semi fusionadas con otras, trajeron profundas transformaciones en las últimas décadas en todos los campos donde la mujer y el hombre se desenvuelven, desarrollando el proceso social de la globalización que hoy día vivimos ${ }^{(1)}$.

Estos cambios, han influenciado también al sector Salud de los diversos países; entre ellos a Chile; planteando la necesidad en ellos de efectuar reformas para mejorar la calidad de la atención en salud, dar respuestas a las expectativas de la población y resolver los problemas sanitarios pendientes ${ }^{(2-3)}$.

Las nuevas demandas de atención en salud originan nuevas acciones y, Enfermería como práctica social encuentra respuestas a las necesidades de cuidado de la salud individual y colectiva buscando mejorar la calidad de vida de las personas ${ }^{(4)}$.

El Departamento de Enfermería de la Universidad de Concepción, se encuentra comprometido frente a los desafíos de este nuevo mundo globalizado, evaluando permanentemente las currículas de pre y postgrado para responder a las necesidades emergentes de esta profesional y de la propìa disciplina.

La carrera de Enfermería de la Universidad de Concepción, en la ciudad de Concepción, en el centro sur del país Chile, fue creada en 1947. En un inicio contempló un curriculum de tres años, y a través de sucesivas evaluaciones necesarias por los cambios epidemiológicos del país y las necesidades de crecimiento del propio colectivo, se llega al actual currículum de la carrera, cuya revisión y aprobación fue sansionada por las autoridades universitarias en el año $2000^{(5)}$.

La primera cohorte de egresados con el nuevo currículum se graduará a fines del 2004, los cuales después del 4 año de estudios, obtienen el grado de Licenciado/a en Enfermería, y al término del 5 año obtienen el título profesional de Enfermera/o que les permite ejercer legalmente la profesión. Actualmente se cuenta con una matrícula de 525 alumnos en pregrado, con una media de 105 alumnos por curso. Su planta académica equivale aproximadamente a 36 jornadas completas ${ }^{(6)}$.

En el año 1982, concientes de la necesidad de profundizar en áreas clínicas específicas y de resolver problemas propios de la profesión, el Departamento de Enfermería comienza la apertura de una serie de Especialidades en Enfermería que hoy día son 6: Especialidad en Enfermería Pediátrica, Neonatología, Unidad de Cuidado Intensivo, Geriatría, Oftalmología, y Diálisis y trasplante renal ${ }^{(7)}$.

En 1980; bajo la conducción de la profesora Srta. María Figueroa F.; el Departamento de Enfermería de la Facultad de Medicina de la Universidad de Concepción, inicia su programa de Magister en Enfermería con las menciones de: Enfermería en Salud Comunitaria y Enfermería Médico-Quirurgica, otorgando un impulso significativo a la investigación en Enfermería en Chile, adquiriendo con ello un espacio indiscutido en los países de habla hispana, siendo un polo importante de desarrollo, lo cual permitió llevar a la Universidad de Concepción al liderazgo nacional en lo que a nuestra disciplina se refiere, tanto a nivel de pregrado como de postgrado ${ }^{(8)}$.

En el año 2000; y bajo la dirección de la suscrita; el programa sufre modificaciones producto de los cambios epidemiológicos del país, de la gran oferta de grados académicos de magister de otros países y, de la necesidad de dejar claramente establecido las diferencias en los diversos niveles de postgrado (magister y doctorado). Además de otorgar un mayor tiempo al análisis reflexivo, a la discusión crítica y a la actividad de investigación en el programa. La Escuela de Graduados de la Universidad, a su vez, se encontraba en una campaña de modernización curricular de los diversos programas de postgrado de la universidad, considerando criterios nacionales que definen estos programas así como estándares de calidad para insertarlos con mayor facilidad dentro del mercado expectante ${ }^{(9)}$. Es así, como se origina el actual programa de Magister en Enfermería de nuestra casa de estudios que dá respuesta a los requerimientos tanto de ambientes internos, como externos al departamento.

\section{PROGRAMA MAGISTER EN ENFERMERÍA}

El programa de Magister en Enfermería, propicia el estudio de la disciplina desde el punto de vista de sus fundamentos filosóficos y teóricos que sustentan el quehacer de Enfermería y las prepara para que se integren a equipos multidisciplinarios de investigación en salud y al desarrollo nacional en el área de su competencia. Además satisface las necesidades de educación permanente que todo grupo profesional tiene.

El objetivo general del programa es preparar 
profesionales capaces de contribuír al desarrollo de la disciplina de Enfermería y, de conducir la docencia, investigación y ejercicio profesional en el país, con un alto grado de competencia.

El alumno egresado del programa es capaz de evaluar la atención de enfermería en el contexto de las metas de salud del país, relacionar la multifactorialidad de los problemas de salud que pueden afectar a los individuos y comunidades, liderar el Proceso de Enfermería con cierto grado de predicción y control, así como tomar decisiones en el ámbito de su competencia. Realizar investigaciones con la asesoría de su profesor guía, formular un marco referencial que integre valores personales y aquellos que identifican la profesión de enfermería aceptada por la sociedad ${ }^{(10)}$.

El programa actualmente vigente consta de un grupo de asignaturas básicas obligatorias y electivas de especialidades que apoyan la tesis que desarrollan durante el 2 año, bajo la orientación de un profesor guía acreditado como tal ante la Escuela de Graduados de la Universidad. Además participan en seminarios con los diversos profesores visitantes invitados al programa.

Su diseño curricular se basa en una estructura entregada por la instancia rectora de los programas de postgrado de la casa de estudios, el cual es similar para todos los programas de postgrado de las diversas facultades de la universidad.

\begin{tabular}{ll} 
Asignaturas Básicas Obligatorias & \\
- Bioestadística para las Ciencias de la Salud I & 3 créditos \\
- Bioestadística para las Ciencias de la Salud II & 3 créditos \\
- Fundamentos Teóricos para el Trabajo & 4 créditos \\
Comunitario de Enfermería & \\
- Fundamentos Teóricos en Salud del Adulto & 4 créditos \\
*- Fundamentos Teóricos en Enfermería & 3 créditos \\
*- Investigación Cuantitativa en Enfermería & 3 créditos \\
Asignaturas Electivas de Especialidad & \\
(De éstas debe escoger mínimo 4 créditos) & \\
- Salud y Mujer & 2 créditos \\
- Satisfacción en el Trabajo & 2 créditos \\
- Enfermería Oncológica & 2 créditos \\
- Sexualidad Humana & 2 créditos \\
- Enfermería en Gerontología & 2 créditos \\
- Autocuidado y Envejecimiento Saludable & 2 créditos \\
- Promoción de la Salud y Prevención de Consumo & 2 créditos \\
de Drogas & \\
*- Abordajes en Torno al Trabajo Femenino & 3 créditos \\
*- Mujer un recorrido por su Salud & 4 créditos \\
*- Enfermería y Calidad de Vida de la Mujer con & 3 créditos \\
Cáncer & \\
*- Enfermería y Salud Mental de la Mujer & 3 créditos \\
*- Análisis Crítico de la Disciplina de Enfermería & 3 créditos \\
Seminarios optativos (con profesores visitantes) & 1 a 2 créditos \\
Proyecto y Desarrollo de Tesis & No tiene créditos \\
Defensa Pública de Tesis & \\
Tiempo Total de duración del Programa: 2 años & \\
\hline
\end{tabular}

El alumno que opta por la modalidad de part-time, tiene un máximo de 3 años para dar término al programa

*asignaturas convalidables para el programa de Doctorado en Enfermería

1 crédito = equivale a 1 hora teórica semanal de clase o 2 horas de laboratorio durante 16 semanas
La modalidad de entrega de las asignaturas es presencial, pudiendo la tesis desarrollarse en la ciudad o país de origen a través de internet y con reuniones presenciales cada vez que sea necesario, siempre bajo la tutoría de su profesor guía.

Las tesis apuntan a las líneas de investigación del programa y son aquellas que tienen los profesores guías, y que se enuncian a continuación:

Líneas de Investigación del Programa de Magister

- Enfermería en Gerontología

- Promoción de la Salud y Prevención del consumo de Drogas

- Sexualidad Humana y Enfermedades Sexuales Transmisibles

- Enfermería y Salud Laboral

- Enfermería y Salud Integral de la Mujer

- Disciplina de Enfermería

El Programa de Magister en Enfermería se encuentra acreditado ante el Consejo Nacional de Postgrado del Ministerio de Educación. Su última acreditación fue en Mayo del año $2002^{(11)}$. A la fecha se han graduado 80 alumnos, provenientes desde Argentina, Chile, Costa Rica, Cuba, Panamá, y Perú. Existen actualmente 11 matriculados, que se encuentran en distintas etapas del proceso.

Los profesores del programa son 13 enfermeras, 1 estadístico, 1 antropóloga y 2 sociólogos quienes asesoran las tesis de grado en los aspectos metodológicos y estadísticos. Todos ellos contemplan solo una jornada parcial para el programa, que va desde 4 a 12 horas máximo semanal. Este cuerpo académico se encuentra apoyado por un grupo de profesores visitantes de diversos países que entregan conocimientos respecto a temas de interés para la disciplina e investigación entre otros ${ }^{(10-11)}$.

Algunos de los impactos del programa se reflejan en una mejor preparación de los alumnos en cuanto la disciplina de Enfermería. En su mayoría, estos alumnos provienen del mundo académico lo que trae consigo un efecto multiplicador, ya que ellos a su vez, son profesores en las distintas escuelas de enfermería en sus respectivos países. Por otro lado ha permitido aumentar la cobertura de las investigaciones en enfermería en forma exponencial respecto a las décadas anteriores, aunque sea necesario aumentarla aún mucho mas. Esto ha traído aparejado ciertamente un aumento de las publicaciones; que siempre son pocas respecto a otras disciplinas que tienen años de historia y creación; pero que ha significado para las 
enfermeras realmente un gran esfuerzo para posicionar la disciplina junto a las demás en los medios de difusión ${ }^{(12)}$.

Por otro lado, el mundo académico universitario ha comenzado a entender que Enfermería es una disciplina emergente, a la cual se le debe otorgar los mismos espacios y oportunidades que a las demás disciplinas y ciencias que se imparten en las universidades. Es en la última década donde ha tenido una mayor socialización y visibilización dentro de la comunidad universitaria, estando aún muy lejos de ocurrir el mismo proceso en la sociedad.

El programa ha tenido también un impacto en el autoestima de las enfermeras/os, ya que les ha permitido a sus egresados ser mas competitivos en el mundo globalizado de hoy, haciendo que éstos puedan continuar estudios de doctorado en otros países, tales como Brasil, Estados Unidos (USA), Canadá, España y por supuesto Chile, con bastante éxito, y asumiendo posteriormente posiciones de liderazgo en los diversos países ${ }^{(12)}$.

En la última década, debido al aumento de las investigaciones, ha existido un incremento considerable de participación de las enfermeras en comunicaciones en los diversos eventos científicos, teniendo que concursar muchas veces entre sí por los fondos disponibles que siempre son escasos. Una buena cantidad de académicos cuyos trabajos son aceptados en congresos, seminarios, jornadas, conferencias u otros, no tienen posibilidad de presentar sus trabajos por falta total o parcial de financiamiento, lo que es una gran pérdida de escenario para la presentación y discusión de los resultados de las producciones científicas, así como de la presencia de las instituciones a la cual representan.

Otro impacto del programa ha sido que sus profesores han sido reconocidos nacional $e$ internacionalmente, lo que les ha otorgado tribuna en diversas instancias y plataformas, como en comités editoriales de revistas, en convenios, ser profesores visitantes o invitados, ser requeridos como expertos, ser asesores en centros de investigación entre otros, lo que ha permitido una mayor socialización de nuestros programas, como de la universidad.

La gran fortaleza que tiene el programa es sin lugar a dudas, la motivación que tienen los propios académicos por irse superando, a pesar que la mayoría de las veces esto signifique un gran recargo de trabajo que va mas allá del horario contratado por la institución.

La debilidad que ha persistido durante todos estos años, tiene que ver con la falta de financiamiento de las investigaciones de las enfermeras de parte de las instituciones ya sean éstas públicas o privadas y, que otorgan fondos para estos fines, porque no logran visualizar la importancia de la creación del conocimiento de enfermería en la salud de las comunidades ${ }^{(12-13)}$. Me permito señalar que es necesario una mayor generosidad de las instituciones relacionadas con salud, hospitales, clínicas, laboratorios; de las empresas, de instituciones de servicios como bancos, financieras, agencias de publicidad entre otras, para que aporten con fondos para desarrollar investigaciones que permitan descubrir como ir mejorando el cuidado de la salud de los individuos, grupos y comunidades con la finalidad de otorgarles una mejor calidad de vida en un mundo tan tecnificado como el de hoy.

\section{PROYECTO DOCTORADO EN ENFERMERÍA}

En el año 1992, conscientes que la preparación de los académicos debía llegar al mayor nivel posible para lograr los máximos aportes a la disciplina, algunos académicos del Departamento de Enfermería, bajo la dirección de Srta. Jasna Stiepovich Bertoni, elaboraron un proyecto que contemplaba la formación de una masa crítica de académicos a nivel de doctorado, con el propósito de abrir posteriormente el primer programa de Doctorado en Enfermería en Chile en la Universidad de Concepción.

Para la preparación de los docentes, el país escogido fue Brasil, y la Universidad, la de Sao Paulo en la ciudad de Ribeirao Preto, debido a la calidad de sus programas y la preparación de sus académicos, así como la mayor cercanía cultural entre los países, lo que permitía prever a futuro el éxito de esta iniciativa.

En el año 1995 se firma un convenio entre las respectivas carreras de enfermería de ambas universidades $^{(14)}$, que entre otras cosas permite dar comienzo a los estudios de doctorado de las académicas. Desde la idea inicial hasta la concreción del inicio de la primera etapa del proyecto pasaron 4 años. Este proyecto contó con el patrocinio de la Rectoría de la Universidad de Concepción y el auspicio en sus diversas etapas, de la Facultad Medicina, del Departamento de Enfermería y, de la fundación Kellog.

Es así como durante el segundo semestre de 1995, los académicos que se motivaron por continuar estudios superiores, iniciaron el programa con dos 
asignaturas de nivel doctorado de una facultad de la propia Universidad de Concepción, que posteriormente fueron oficialmente convalidadas por el programa de Doctorado en Enfermería (Interunidades) de la Universidad de São Paulo en Ribeirão Preto, Brazil ,como asignaturas de tipo electivas.

En Marzo de 1996, 4 académicas parten a la ciudad de Ribeirao Preto en Brasil, a la universidad escogida, a cursar el programa. Durante ese semestre se cursaron las asignaturas obligatorias además de otras electivas. En el semestre siguiente dos profesoras del programa vinieron a Chile a dictar asignaturas. Asistieron además a los cursos, alumnas brasileras del programa que se trasladaron a nuestro país. Se continuó posteriormente con el proyecto de tesis y el desarrollo de éste a través de internet y viajes a la universidad en Brazil, o de los profesores orientadores de tesis a nuestro país, cuando fuere necesario.

Es así que, durante el primer semestre de 1999 obtienen sus grados de doctoras en Enfermería las Sras/ Srtas. Tatiana Paravic Klijn, Olivia Sanhueza Alvarado, Jasna Stiepovich Bertoni y Sandra Valenzuela Suazo, siendo ellas las primeras doctoras en la disciplina de la Universidad de Concepción, habiendo solo una doctora en Enfermería mas en todo el país en esa fecha, perteneciente a otra casa de estudios superiores.

Así culmina la primera parte del proyecto, en los tiempos establecidos por ambas universidades, dando respuesta en forma responsable a las expectativas depositadas en estas académicas.

La Dra. Jasna Stiepovich Bertoni fue nombrada Sub-Directora de la Escuela de Graduados (postgrado) de la Universidad de Concepción y la Dra. Tatiana Paravic Klijn directora del Postgrado del Departamento de Enfermería.

En Agosto de 1999 se comienza con la segunda etapa del proyecto, que es la creación de un programa de Doctorado en Enfermería para la Universidad de Concepción, desafío que es asumido por las doctoras en Enfermería Sras. Sandra Valenzuela, S.; Olivia Sanhueza A. y Tatiana Paravic, K. bajo la dirección de ésta última, realizando la confección del programa con todas las asignaturas, sus líneas de investigación, la reglamentación y la fundamentación de la necesidad de la apertura de un programa en la disciplina de Enfermería en el país.

El proyecto es terminado a fines de ese año y presentado por la suscrita ante la Asamblea de docentes del Departamento de Enfermería quienes se informan en detalle de ello, entregando algunos alcances que son incorporados al proyecto, quedando éste listo para proseguir la etapa siguiente que fue presentarlo al Consejo de Graduados de la Facultad de Medicina, conformado por enfermeras y matronas con grado académico de magister y por médicos especialistas, representantes de los diversos programas de especialidades de medicina, matronería y enfermería. Este consejo estimó necesario hacer algunos cambios al proyecto por lo que vuelve a la comisión formada por las doctoras en Enfermería, donde se estudia y se incorporan las sugerencias. En una segunda presentación del programa a este consejo es aprobado por éste, pasando ahora a la instancia superior que es el Consejo de Facultad de Medicina, integrado por los directores de los diversos departamentos de la facultad de medicina, la directora de enfermería, la directora de las matronas, mas algunos consejeros académicos y, las autoridades de la facultad, tales como el decano, vicedecano, y secretario académico.

Algunos médicos y las matronas integrantes de este consejo, en su totalidad se opusieron al proyecto entregando un sinfín de razones de toda índole, por lo que nuevamente vuelve el proyecto a sus bases. Se analizan las sugerencias, y se estudian las razones de la no aprobación y las estrategias que permitirían aprobarlo en una próxima oportunidad. Se realiza un estudio de las diversas investigaciones realizadas por las enfermeras académicas durante los últimos 20 años, ya que la limitada producción del conocimiento y la escasa captación de recursos financieros, fue la razón de mayor peso aducida por los médicos, para no aprobar el proyecto de doctorado. Muy pocos de ellos conocían los trabajos de investigación de las enfermeras. Por otra parte, se realizó un lobby entre algunos de los médicos que integraban este consejo, para una mayor sensibilización respecto al proyecto.

Es así que algunos meses después se presenta el proyecto por segunda vez ante este consejo, iniciando el relato con la historia del nacimiento y desarrollo del programa de Magister en Enfermería que ya llevaba 22 años ininterrumpidos y, la producción científica de los académicos durante los últimos 20 años, así como la fundamentación, tanto para la disciplina como para el país, de abrir un programa de Doctorado en Enfermería, presentando al final el programa con sus asignaturas. En esta ocasión fue aprobado por simple mayoría, a pesar de votos en contra (con una sola exepción) de las matronas y de unos pocos médicos. 
Una vez cumplidos todas las etapas a nivel de facultad, el proyecto sale hacia la Escuela de Graduados que es la instancia rectora de los programas de postgrados donde éste debe ser sansionado por su Consejo. En esta instancia permanece por un año antes de ser presentado a Evaluadores Externos Internacionales, quienes fueron escogidos de dos universidades brasileras, con trayectoria en postgrado y en las líneas de investigación del programa a evaluar. Estas universidades fueron las de São Paulo, Ribeirão Preto y Rio de Janeiro(15).

Los informes de los evaluadores fueron altamente positivos, aspecto que permite presentar entonces el programa al Consejo de Graduados de la Universidad, integrado por los jefes de los programas de magister y doctorados de todas las facultades de la universidad.

Esta etapa del proceso fue quizás la mas difícil de sortear, ya que varios de los consejeros no veían a la carrera de Enfermería como una disciplina que debería tener un programa de doctorado. Nuevamente se hicieron sugerencias al programa que hubo que integrarlas antes de ser presentado por segunda vez a este Consejo.

Se realizó nuevamente un lobby con algunos de estos consejeros para entrar a explicar porqué se creía que Enfermería merecía un espacio y una oportunidad en el mundo de hoy. Finalmente fue aprobado por este consejo en Junio 2002.

El director de la Escuela de Graduado consideró que era necesario un tiempo de espera antes que él presentara el programa al Consejo Superior de la Universidad, última etapa en todo este proceso. Finalmente, meses después, fue aprobado por decreto $\mathrm{n}^{\circ}$ 2003-06, del 7 de Marzo $2003^{(16)}$.

La primera convocatoria del Programa de Doctorado en Enfermería de la Universidad de Concepción, que es además el primero del país, se abrió en Mayo del presente año hasta $1^{\circ}$ de Diciembre 2003, con un cupo de 5 alumnos para este primer curso. El programa iniciará sus actividades académicas en Marzo del 2004, bajo la dirección de la Dra. Sandra Valenzuela S.

\section{PROGRAMA DE DOCTORADO EN ENFERMERÍA}

\section{Este programa surge como:}

- una necesidad de la disciplina de construir marcos conceptuales y teóricos, cuyos delineamientos estuviesen de acuerdo con la realidad latinoamericana, con enfoques que sustenten sus bases en el individuo, familia y comunidad y donde la relación salud/enfermedad/contexto/ desarrollo-humano y participación social sean sus pilares fundamentales;

- un programa que propone responder a los desafíos del desarrollo científico y tecnológico actual creando espacios y relaciones horizontales para fortalecer el trabajo en equipos interdisciplinarios y transdisciplinarios;

- una necesidad de aumentar considerablemente el número de enfermeras investigadoras con una base científica, ética y humanista, dotándolas de una conciencia crítica de manera que les permita actuar responsablemente frente a los requerimientos y tendencias del mundo contemporáneo y liderar creativamente procesos de cambio;

- un espacio de análisis de los problemas nacionales de enfermería y proponer, con independencia, formulaciones y soluciones pertinentes ${ }^{(17)}$.

El Programa busca no sólo satisfacer una necesidad académica de los profesionales de enfermería del país, sino que contribuir con sus aportes al desarrollo del conocimiento de la disciplina y a la resolución de problemas propios de la salud de la población, percibiéndose además como el paso inminente en la evolución de los Programas de Postgrado.

El compromiso social de Enfermería hace que ésta mire críticamente la situación en que le corresponde actuar, buscando alternativas creativas relacionadas con el proceso optimizador de las respuestas a las necesidades de salud y otras necesidades derivadas de la naturaleza multidimensional del ser humano, con el desafío de entregar cuidados de salud, conjugando equidad,

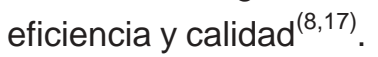

El propósito general del Programa de Doctorado en Enfermería es formar investigadores independientes, altamente calificados que puedan dar respuestas a las interrogantes derivadas del ser, quehacer y saber de la disciplina de Enfermería.

El egresado del programa es una persona que desarrollará la capacidad para realizar investigaciones independientes, el pensamiento crítico y la capacidad analítica. Sabrá utilizar la información científica existente; y será una persona innovadora y creativa que podrá ejercer un liderazgo transformativo. Asimismo se espera de ella un compromiso para abogar y defender los derechos de la persona en la atención de salud de calidad. Sus conductas responderán a una ética cultural y socialmente aceptable $^{(17)}$. 
Plan de estudios.

El candidato a grado de Doctor/a en Enfermería deberá aprobar al menos 33 créditos totales

totas en el desarrollo del programa. De los 33 créditos mínimos:

- 14 créditos corresponderán a asignaturas básicas

- 19 créditos corresponderán a asignaturas electivas de especialización

- y Seminarios adicionales.

\section{Asignaturas Básicas Obligatorias}

- Bioestadística avanzada I

- Fundamentos Teóricos de Enfermería

- Problemáticas de Enfermería

Asignaturas Electivas de Especialización

- Mujer: un recorrido por su salud

- Abordajes en torno al trabajo femenino

- Enfermería y calidad de vida de la mujer con cáncer

- Enfermería y Salud mental de la mujer

- Ergonomía y trabajo de Enfermería

- Mujer, actividad física y calidad de vida

- Análisis crítico de la disciplina de Enfermería

- Bioestadística Avanzada II

- Investigación cualitativa en Enfermería

- Seminario de investigación cuantitativa

- Seminario de investigación cualitativa

Seminarios optativos (con profesores visitantes)

Proyecto desarrollo de tesis

Defensa Pública de Tesis

El alumno podrá tomar asignaturas de otros programas de nivel doctorado, tanto a nivel nacional como internacional, debidamente autorizado por el profesor guía de tesis y el comité asesor de programa, siempre que estos profundicen o complementen el desarrollo de la tesis.

Tiempo de duración del programa es de 3 años a tiempo completo. El tiempo maximo contemplado para este programa es de 5 años, para aquellos alumnos que deseen cursarlo con la modalidad de part-time.

Líneas de Investigación del Programa de Doctorado

- Enfermería y Salud Laboral

- Enfermería y Salud Integral de la Mujer

- Enfermería como Disciplina

Se espera que los conocimientos generados a partir de estas investigaciones se proyecten a nivel de las diversas políticas vigentes, las múltiples acciones en los niveles técnicos, las transformaciones institucionales, la calidad de vida de las comunidades, y el aumento de conocimiento de la disciplina de Enfermería ${ }^{(17)}$.

El núcleo estable de profesores son académicos con grado de doctor y acreditados como tales ante la Escuela de Graduados, pertenecientes a las disciplinas de Enfermería, Psicología, Sociología, Ergonomía, Educación Física y Medicina, pertenecientes a la Universidad de Concepción, además de un grupo de profesores visitantes de otras universidades, tanto nacionales como internacionales.

Desde que se gestó la idea de abrir un Programa de Doctorado en la Disciplina, tener una masa crítica de académicas y aprobar el proyecto en las diversas instancias de la universidad hasta el inicio de éste, han pasado 11 años, no exento de muchas dificultades e incomprensiones $^{(18)}$, pero que con trabajo y la fuerza de la motivación, permitió coronar con éxito la creación de este programa que marca un hito en la historia de la Enfermería Chilena.

\section{CONCLUSIONES}

El postgrado stricto sensus en Enfermería, se ha desarrollado en Chile con mucho esfuerzo, motivación y perseverancia de algunos académicos de la Universidad de Concepción, que tuvo durante 22 años el único Programa de Magister en Enfermería en el país, así como el primer Programa de Doctorado en Enfermería aprobado recientemente y que comienza con su primer curso en Marzo 2004. Estos avances significativos permiten mirar los escenarios futuros, cambiantes y competitivos de este mundo globalizado, con una mirada desafiante y cargada de optimismo.

\section{AGRADECIMIENTOS}

Agradecimientos especiales a aquellas profesoras y mujeres que en forma desinteresada:

- crearon y dieron origen al primer programa de Magister en Enfermería en Chile.

- tuvieron un papel destacado en alguna etapa en el largo proceso que culminó en la creación del primer programa de Doctorado en Enfermería en Chile.

A todas las profesoras del programa de Magister en Enfermería de la Universidad de Concepción. Chile.

A todas las autoridades de la Escuela de Enfermería de la Universidad de São Paulo en Ribeirão Preto, Brasil, y muy en especial a las profesoras del programa Interunidades del Doctorado en Enfermería de dicha Universidad, que nos tendieron una mano cuando lo necesitábamos.

A todas aquellas personas que anónimamente hicieron posible nuestro sueño. 


\section{REFERENCIAS BIBLIOGRÁFICAS}

1 .Godué CH. La salud en los procesos de globalización y de internalización. In: Grupo Salud Internacional Pontificia Universidad Javeriana. Relaciones internacionales, política social y salud. Desafíos en la era de la globalización. Bogotá; 1998. p.25-45.

2. Ahumada C. Política Social y Reforma de Salud en Colombia. In: Facultad de Ciencias Políticas y Relaciones Internacionales Pontificia Universidad Javeriana. Papel Político \# 7. Bogotá; 1998. p.9-35.

3. Ministerio de Salud(Chile). Los objetivos sanitarios para la década 2000-2010. (Chile): Ministerio de Salud. División de Rectoría y Regulación Sanitaria. Departamento de Epidemiología; 2002.

4. Paravic T. Prioridades de investigación en Enfermería para la reducción de la demanda de las drogas. VI Reunión Internacional del proyecto piloto de Enfermería para América Latina en promoción de la salud, prevención y tratamiento del uso y abuso de drogas y reinserción social. CICAD/OEA; 2001 junio 25-28;Washington; 2001.

5. Universidad de Concepción (Chile). Documento oficial. Decreto \# 2000-09. Concepción; 2000.

6. Universidad de Concepción (Chile). Departamento de Enfermería. Registros oficiales. Concepción; 2003.

7. Jofré V. La situación de los programas de especialización en Enfermería en Chile. In: Situación de los programas de postgrado de Enfermería en nueve países de América Latina frente a los desafíos de la reducción de la demanda de drogas. CICAD/OEA; 2003. p.71-4.

8. Departamento de Enfermería Universidad de Concepción. Proyecto Doctorado en Enfermería. Documento oficial. Concepción; 2003.

9. Departamento de Enfermería Universidad de Concepción. Autoevaluación de programa de Magister en Enfermería para su Acreditación. Documento oficial. Concepción; 2002.

10. Departamento de Enfermería Universidad de Concepción. Programa Magister en Enfermería Documento oficial. Concepción; 2000.

11. Consejo Nacional de Postgrado del Ministerio de Educación. Notificación oficial de Acreditación del Programa Magister en Enfermería. 2002.

12. Paravic T. La situación del programa de Magister en Enfermería del Departamento de Enfermería Universidad de Concepción. Documento para consolidado de la evaluación de los programas de postgrado de Enfermería en América Latina. CICAD/OEA. Concepción; 2002. p.1-23.

13. Lenise M, Lima F. Producción de conocimiento en Enfermería en Latinoamérica: El Estado del Arte. Reunión Internacional. Belo Horizonte; Minas Gerais; Brasil. Agosto 27-1 Septiembre. 2000. p. 1-51.

14. Universidad de Concepción, Universidad de Sao Paulo. Convenio entre respectivas carreras de enfermería .Documento oficial. 1995.

15. Escuela de Graduados Universidad de Concepción. Evaluación de pares externos del programa de Doctorado en Enfermería. Documento oficial. 2002.

16. Universidad de Concepción. Documento oficial. Decreto 2003-06. 2003.

17. Departamento de Enfermería Universidad de Concepción. Programa Doctorado en Enfermería. Documento oficial. Concepción; 2003.
18. Paravic T, Valenzuela S. Proyecto programa de Doctorado en Enfermería. Departamento de Enfermería. Universidad de Concepción; Concepción. Chile. In: Situación de los programas de postgrado de Enfermería en nueve países de America Latina frente a los desafíos de la reducción de la demanda de drogas. CICAD/OEA. Concepción; 2003. p. 714. 